\title{
Chronic psychosocial stress causes delayed extinction and exacerbates reinstatement of ethanol-induced conditioned place preference in mice
}

\author{
Amine Bahi • Jean-Luc Dreyer
}

\begin{abstract}
Rationale We have shown previously, using an animal model of voluntary ethanol intake and ethanol-conditioned place preference (EtOH-CPP), that exposure to chronic psychosocial stress induces increased ethanol intake and EtOH-CPP acquisition in mice.

Objective Here, we examined the impact of chronic subordinate colony (CSC) exposure on EtOH-CPP extinction, as well as ethanol-induced reinstatement of CPP.

Methods Mice were conditioned with saline or $1.5 \mathrm{~g} / \mathrm{kg}$ ethanol and were tested in the EtOH-CPP model. In the first experiment, the mice were subjected to 19 days of chronic stress, and EtOH-CPP extinction was assessed during seven daily trials without ethanol injection. In the second experiment and after the EtOH-CPP test, the mice were subjected to 7 days of extinction trials before the 19 days of chronic stress. Druginduced EtOH-CPP reinstatement was induced by a priming injection of $0.5 \mathrm{~g} / \mathrm{kg}$ ethanol.

Results Compared to the single-housed colony mice, CSC mice exhibited increased anxiety-like behavior in the elevated plus maze (EPM) and the open field tests. Interestingly, the CSC mice showed delayed EtOH-CPP extinction. More importantly, CSC mice showed increased alcohol-induced reinstatement of the EtOH-CPP behavior.

Conclusion Taken together, this study indicates that chronic psychosocial stress can have long-term effects on EtOH-CPP extinction as well as drug-induced reinstatement behavior and may provide a suitable model to study the latent effects of
\end{abstract}

\footnotetext{
A. Bahi $(\bowtie)$

Department of Anatomy, Tawam Medical Campus,

College of Medicine and Health Sciences, United Arab Emirates

University, PO Box 17666, Al Ain, UAE

e-mail: amine.bahi@uaeu.ac.ae

J.-L. Dreyer

Division of Biochemistry, Department of Medicine,

University of Fribourg, Fribourg, Switzerland
}

chronic psychosocial stress on extinction and relapse to drug abuse.

Keywords Chronic psychosocial stress $\cdot$ CPP · Ethanol · Extinction $\cdot$ Reinstatement $\cdot$ Relapse

$\begin{array}{ll}\text { Abbreviations } \\ \text { CPP } & \text { Conditioned place preference } \\ \text { CSC } & \text { Chronic subordinate colony } \\ \text { EPM } & \text { Elevated plus maze } \\ \text { EtOH } & \text { Ethanol } \\ \text { OF } & \text { Open field } \\ \text { SHC } & \text { Single-housed colony }\end{array}$

\section{Introduction}

Alcohol is one of the most commonly abused substances. However, the etiology of alcohol dependence remains poorly elucidated and only a few treatments are available [for review see Heilig and Egli 2006]. In addition, one of the most troubling aspects of ethanol abuse and alcoholism is the relapse that may occur after several years of abstinence. Several reports have shown that, when exposed to stressful events, humans are more likely to relapse to ethanol and other drugs (Chaplin et al. 2010; Fox et al. 2005; Kosten et al. 1986; Sinha et al. 2000). A factor long known to increase the risk for development and maintenance of alcohol use disorders is environmental stress (Zimmermann et al. 2004). Environmental stress may produce an exaggerated endocrine response, which is normalized by alcohol, often in conjunction with genetic risk factors for alcoholism (Sinha 2001, 2007). Nevertheless, the biological mechanisms by which chronic stress increases craving and relapse to chronic alcohol abuse are unclear. One potential mechanism is that stress acts by altering 
the pharmacological and subjective effects of alcohol. Stress produces a cascade of physiological and psychological effects, each with a distinctive time course. In turn, alcohol administration also influences both ACTH and cortisol secretion (Zimmermann et al. 2004). Thus, there are bi-directional relationships between alcohol and stress: alcohol influences responses to stress, and stress changes reactions to alcohol, depending on an individual's pattern of response to alcohol (Childs et al. 2011). Alcohol's anxiolytic effect is probably another important underlying mechanism of abuse that may induce reinforcement of abuse habits by reduction of negative feelings and negative self-perception, most probably leading to a repetition effect resulting in habituation, although the dampening of stress hormone response by alcohol does not appear to be paralleled by any effect on how subjects perceived the stress situation (Zimmermann et al. 2004).

Chronic stress in humans enhances the susceptibility for addiction disorders including alcoholism (Enoch 2011; Lipton 1997). This has been recapitulated in animal models, but most studies have utilized non-social rather than social stress paradigms, of which the latter are believed to be more relevant to the human situation, mainly for a lack of appropriate animal models. Nevertheless, recent studies have found that chronic psychosocial stress in mice induces an anxiogenic-like phenotype and enhances alcohol consumption and preference (Bahi 2013a; Peters et al. 2013) as well as ethanol-induced conditioned place preference (EtOH-CPP) (Bahi 2013a). Animal studies reporting a positive correlation between stress and alcohol consumption suggest that drinking may take place in response to chronic stress if it is perceived as unavoidable (Nash and Maickel 1988; Volpicelli 1987), when alternative resources are lacking, when alcohol is accessible, and when the individual believes that alcohol will help to reduce the stress (Jennison 1992). When exposed to unavoidable shock, animals exhibit the hormonal changes indicative of the stress response, including increased levels of corticosteroid hormones (Volpicelli 1987). On the other hand, under certain circumstances and at certain doses, alcohol may induce rather than reduce the body's stress response (Waltman et al. 1993). As a matter of fact, several studies demonstrate that alcohol actually induces the stress response by stimulating hormone release by the hypothalamus, pituitary, and adrenal glands (Krishnan et al. 1991; Tsigos and Chrousos 2002; Wand and Dobs 1991).

In the present study, we aimed to examine whether chronic subordinate colony (CSC) housing-induced chronic psychosocial stress would affect extinction and reinstate ethanolconditioned place preference. We believe that such findings may be of considerable consequence to tackle alcoholism and drug abuse in humans. Indeed, they would suggest that stimuli previously associated with social stressful events could precipitate and accelerate relapse after years of abstinence.

Therefore, in the present study, we examined the effects of chronic psychosocial stress and CSC housing on the extinction of EtOH-CPP, and we investigated the impact of CSC housing on ethanol-induced reinstatement of EtOH-CPP after extinction. Based on preliminary findings from our laboratory and the previously published study (Bahi 2013a, b), we hypothesized that the CSC housing would delay $\mathrm{EtOH}-\mathrm{CPP}$ extinction and exacerbate drug-induced reinstatement after extinction.

\section{Materials and methods}

\section{Animals}

Male C57BL/6 mice weighing 19-24 g (experimental mice) or Tuck-Ordinary "TO" (resident mice) weighing 35-40 g were housed in standard Plexiglas observation cages $(35 \times 23 \times$ $19 \mathrm{~cm}$ ) before the experimental procedure started. All mice were bred in the local central animal facility of the CMHS and were kept under standard laboratory conditions. A bedding was produced locally and autoclaved before use and the mice had free access to tap water and standard mouse chow diet obtained from the National Feed and Flour Production and Marketing Company LLC (Abu Dhabi, UAE). The experimental procedures were approved by the local research ethics committee (protocol no. A01-12).

\section{Drugs}

Ethanol (catalog number 131086) was purchased from Panreac Quimica SAU (Barcelona Spain). For the CPP experiment, ethanol was diluted in isotonic saline $(\mathrm{NaCl} 0.9 \%)(10 \% ; v / v)$ and injected intraperitoneally (IP) at $1.5 \mathrm{~g} / \mathrm{kg}$.

Chronic subordinate colony housing stress procedure

The CSC procedure has been previously described (Bahi 2013a). In brief, four C57BL/6 experimental subordinate "intruder" mice were introduced into the home cage of a larger, dominant TO outbred male mouse "resident" that had been housed with female mice for 10 days to enhance its territoriality and aggression. The CSC procedure lasted for 19 consecutive days. To avoid habituation the four intruder mice were transferred to a novel resident cage on day 8 and 15. In all colonies, the larger male mouse established a dominant status by chasing and attacking all four experimental mice. In parallel, singlehoused control (SHC) mice remained undisturbed in their home cages except for a change of bedding once a week if needed. A total of 48 mice were used $(\mathrm{SHC}=16, \mathrm{CSC}=16)$.

\section{Elevated plus maze test}

At the end of the CSC procedure, the anxiety-like behavior was assessed using the elevated plus maze (EPM) test as described previously between 0800 hours and 1100 hours 
for 5 min (Bahi 2013a, b; Bahi and Dreyer 2012a; Bahi et al. 2009). The EPM apparatus was made of wood painted black and elevated $0.4 \mathrm{~m}$ above the ground. The maze was composed of two open arms $(40 \times 6 \mathrm{~cm})$ aligned perpendicularly to two closed arms $(40 \times 6 \times 30 \mathrm{~cm})$. The open arms had a $2-\mathrm{mm}$ high wood rim to prevent falling. In brief, each mouse was placed on the central platform facing a closed arm and the number of entries into the open and closed arms as well as the time spent on the respective arms were manually recorded to allow calculation of the percentage of time spent on, and the percentage of entries performed into the open arms of the maze. A total of 48 mice were tested $(\mathrm{SHC}=24, \mathrm{CSC}=24)$.

\section{Open field test}

After completion of the EPM test, mice were tested in an open field (OF) for their anxiety-like behavior, and locomotor activity was measured as described previously (Bahi 2013a, b; Bahi and Dreyer 2012a; Bahi et al. 2009). The test was carried out in the OF arena between 1300 hours and 1500 hours $24 \mathrm{~h}$ after the completion of the chronic stress housing. The OF was a $32 \times 32 \mathrm{~cm}$ white Plexiglass arena marked into 64 equal squares by black lines and surrounded by $20-\mathrm{cm}$-high walls. The central 16 squares were defined as the center area. The mice were put in the center of the arena and allowed to explore freely for $10 \mathrm{~min}$. Line-crossing (defined as at least three paws in a square) and the time spent in the center of the arena were manually recorded and used as a measurement of locomotion and anxiety, respectively. In total, 48 mice were used (SHC= $24, \mathrm{CSC}=24)$.

Ethanol-induced conditioned place preference

The CPP apparatus used in the current study has been previously described (Bahi 2012, 2013a, b; Bahi and Dreyer 2012b; Bahi et al. 2013b). In brief, it consists of two equalsized compartments $(30 \times 30 \times 30 \mathrm{~cm})$ with a sliding guillotine door in the center of the base. Both compartments are equipped with different visual and tactile cues: one is black with a large metal grid floor, the other is white with a fine metal grid rod floor. Following an unbiased procedure in terms of initial spontaneous preference, the effects of chronic subordinate colony housing on EtOH-CPP extinction and reinstatement were studied in C57BL/6 mice. The place conditioning consisted of three phases. During the first phase (pre-conditioning, day 1), the mice were allowed to access both chambers of the apparatus for $15 \mathrm{~min}$ and the time spent by the animal in each chamber was recorded during a 900 -s period. During the second phase (conditioning, day 2-9), and before eight daily 30 -min conditioning sessions (four alternating drug and vehicle pairings), the animals received IP injections of ethanol or isotonic saline, and the guillotine door between the two compartments was closed. The mice received ethanol injections $(1.5 \mathrm{~g} / \mathrm{kg}$ prepared from $10 \%$ ethanol solution in isotonic saline $v / v$ ) on drug days and were placed 1-2 min later in one compartment. On non-drug days, these animals received isotonic saline injections $(10 \mathrm{~mL} / \mathrm{kg})$ and were subsequently placed in the opposite compartment. The presentation of ethanol was counterbalanced so that half of the mice were injected with the drug on days $2,4,6$, and 8 , and the other half on days $3,5,7$, and 9. During the third phase (post-conditioning, day 10), the guillotine doors separating the two chambers were removed and the time spent by the mice in each chamber was recorded during a 900 -s test period. The difference in seconds between the time spent in the EtOHpaired compartment in the post- and pre-conditioning tests is a measurement of the degree of conditioning induced by the drug. The detailed procedure has been described previously (Bahi 2013a; Bahi and Dreyer 2012b, c; Bahi et al. 2013a, b).

Place preference to ethanol was extinguished as previously described (Bahi 2012). Mice were injected with isotonic saline and allowed to move freely in the apparatus for $900 \mathrm{~s}$. This procedure occurred for 7 consecutive days to extinguish place preference, i.e., until the mean difference between the time spent in the EtOH-paired and the saline-paired chambers was no longer statistically significant.

Reinstatement was performed the day following extinction. Reinstatement was identical to the post-conditioning test session except that mice were injected with an ethanol prime $(0.5 \mathrm{~g} / \mathrm{kg}, \mathrm{IP})$ for drug-induced reinstatement. Immediately after injection, the mice were placed in the central zone of the CPP apparatus with free access to both chambers. The time spent in each chamber was recorded for $900 \mathrm{~s}$ to enable comparison with the last extinction session.

\section{Experiment 1: effects of chronic psychosocial stress on EtOH-CPP extinction}

After the post-conditioning CPP test, the mice were divided into two groups: a single-housed group (SHC; $n=8)$ and chronically stressed colony-housed mice (CSC; $n=8)$. After 19 days, the EPM and open field tests were performed on day 30 and EtOH-CPP extinction started for 7 days as detailed above. The experimental timeline is depicted in Fig. 1a.

\section{Experiment 2: effects of chronic psychosocial stress on ethanol-induced reinstatement after extinction of EtOH-CPP}

After the EtOH-CPP extinction was determined, experimental mice $(\mathrm{CSC}=8)$ were exposed to 19 days of chronic stress as described above. Control mice were single-housed $(\mathrm{SHC}=8)$. On day 37 , both groups were tested for the anxiety-like behavior and locomotor activity in the EPM and OF tests as 
a

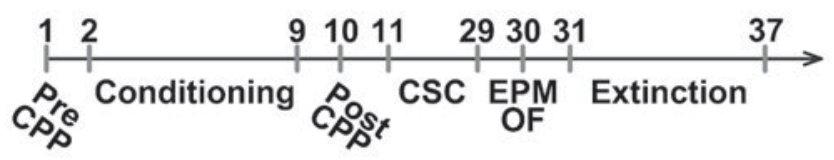

b

${\underset{C}{S_{\delta}^{s}}}_{12}^{2}$

Fig. 1 Schematic representation of the experimental procedures. The timelines show sequence and duration of experimental protocols of the effect of CSC on a EtOH-CPP extinction (Experiment 1), b ethanol-induced reinstatement of EtOH-CPP behavior after extinction (Experiment 2)

described above. Twenty-four hours later, the mice were given a priming injection of ethanol $(0.5 \mathrm{~g} / \mathrm{kg}$, IP) before being placed between the two chambers with access to the entire apparatus for $15 \mathrm{~min}$. The time spent on each side was recorded. The experimental timeline is depicted in Fig. 1b.

\section{Statistical analysis}

For statistical comparisons, the software package SPSS (version 19.0) was used. Data were expressed as means \pm SEM. The analysis of the effects of housing on body weight gain, the elevated plus maze, and the open field behavior were analyzed using one-way analysis of variance (ANOVA). Also, EtOHCPP pre-, post-conditioning, the difference and reinstatement scores (Experiment 2) were analyzed using one-way ANOVA. Finally, the effects of housing on weight gain and EtOH-CPP extinction data were analyzed using one-way ANOVA with repeated measure with housing (CSC or SHC) as the betweensubjects factor and time as the within-subjects factor. The criterion for statistical significance was $p \leq 0.05$.

\section{Results}

Effects of chronic psychosocial stress on body weight gain

As expected, total body weights of the CSC $(n=16)$ group were lower than SHC $(n=16)$. In fact and as depicted in Fig. 2a, oneway ANOVA with repeated measure have shown that there was a main effect of time $\left(F_{(3,90)}=168.306, p<0.0001\right)$ and stress $\left(F_{(1,30)}=8.022, p=0.008\right)$. More importantly, the interaction between stress and time was significant $\left(F_{(3,90)}=11.964\right.$, $p<0.0001)$. In addition and as shown in Fig. $2 \mathrm{~b}$, the weight gain between the first and the 19th day of chronically stressed mice was significant $\left(F_{(1,30)}=26.230, p<0.0001\right)$.
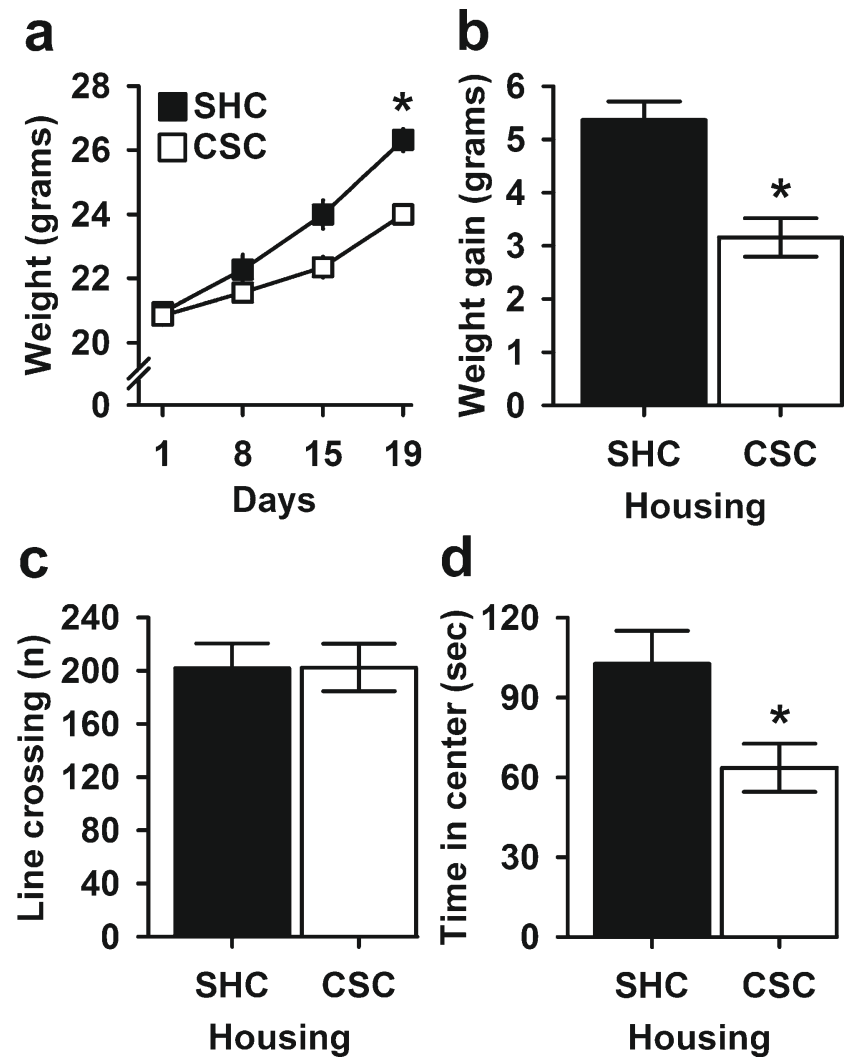

Fig. 2 Chronic psychosocial stress effects on body weight gain and anxiety-like behavior in the open field test. a The daily defeated group CSC male mice gained significantly less body weight compared to singlehoused controls $(\mathrm{SHC})$. b Chronically stressed mice showed attenuated weight after 19 days CSC. c In the OF test, there was no significant difference between the SHC and CSC mice in general locomotor activity, as measured by total line crossings. In contrast, $\mathbf{d}$ CSC mice spent less time in the center of the arena in center time indicating an increased anxiety-like behavior of these mice compared to the SHC. Data represent mean \pm SEM. ${ }^{*} p<0.005$ versus single-housed (SHC) control mice. SHC $(n=16)$ and $\operatorname{CSC}(n=16)$

Effects of chronic psychosocial stress on locomotor activity and anxiety-like behavior

After completion of the chronic psychosocial procedure, CSC $(n=16)$ and SHC $(n=16)$, the mice were assessed in the EPM and $\mathrm{OF}$ tests. The OF test quantifies anxiety-like behaviors and locomotor activity. The mice preferentially move around the periphery of an open arena in a novel environment. Time spent in the central area of the open field is considered to be inversely correlated to their level of anxiety-related proneness (Cunha and Masur 1978; Joffe et al. 1973). Therefore, results have shown that stress had no effect on basic locomotor activity $\left(F_{(1,30)}=\right.$ $0.0003, p=0.985$ ) (Fig. 2c). In contrast and as expected, oneway ANOVA revealed that CSC mice exhibited increased anxiety-like behavior and spent less time in the center of the arena (main effect of stress: $F_{(1,30)}=6.504, p=0.016$ ) (Fig. 2d).

To evaluate the anxiety-like behavior following stress exposure, the EPM test was used. One-way ANOVA did reveal a 
main effect of stress on the time spent in the different arms of the EPM. In detail, CSC mice showed less percentage of time spent in the open arms $(\mathrm{OA})\left(F_{(1,30)}=8.113, p=0.008\right)$ (Fig. 3a). Consequently, the total number of entries into OA was lower in CSC mice $\left(F_{(1,30)}=8.189, p=0.008\right)$ (Fig. 3b). Additionally, the percentage of entries into the OA was less in the CSC mice $\left(F_{(1,30)}=\right.$ 7.666, $p=0.010$ ) (Fig. 3c). More importantly and as depicted in Fig. 3d, the total distance moved within the maze was not affected by the stress as the total number of entries into closed arms was not different between SHC and CSC groups $\left(F_{(1,30)}=\right.$ $0.572, p=0.455)$. Taken together, the CSC mice exhibited more anxiety-like behavior than their SHC control mice, but there were no differences in basic locomotor activity per se.

Experiment 1: effects of CSC housing on EtOH-CPP extinction

In this experiment, mice were conditioned based on a previously established unbiased EtOH-CPP paradigm (Bahi 2013a;
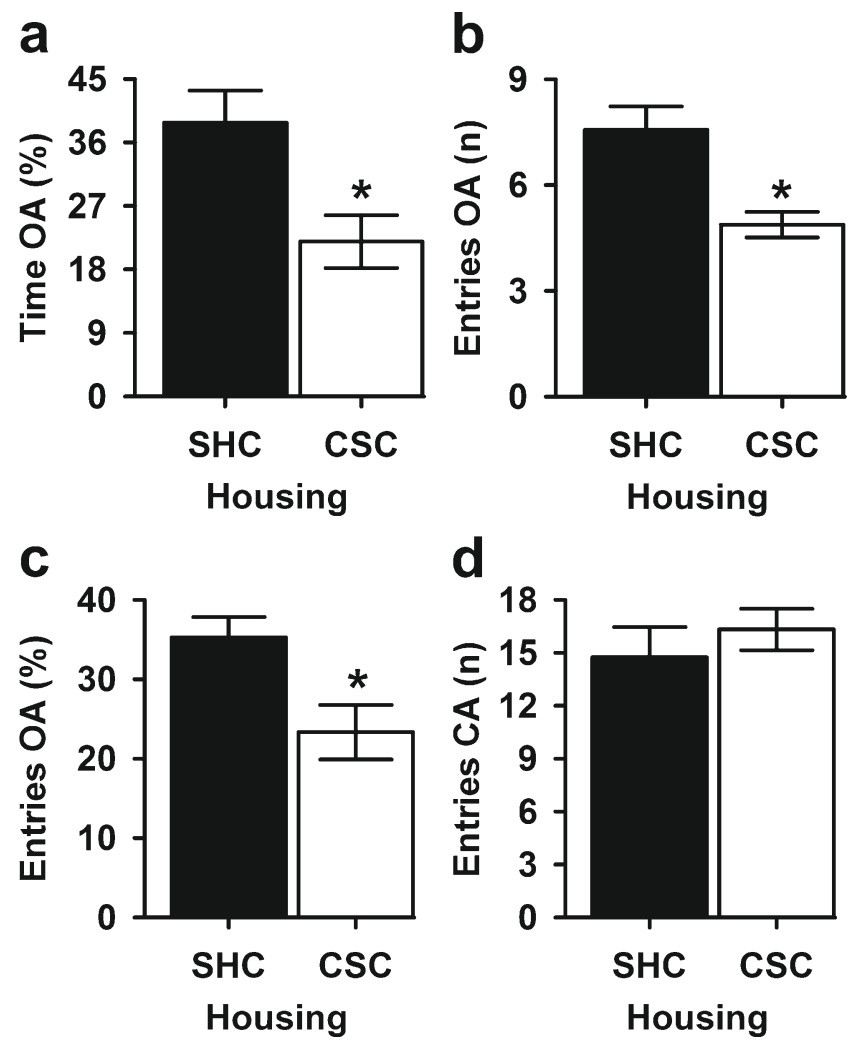

Fig. 3 Chronic psychosocial stress effects on anxiety-like behavior in the elevated plus maze test. Following 19 days of chronic psychosocial stress exposure, CSC mice showed a reduced percentage time in the open arms $(O A)$, b reduced total number of entries into the $\mathrm{OA}$, and $\mathbf{c}$ reduced percentage of entries into the OA of the maze; $\mathbf{d}$ however, both $\mathrm{SHC}$ and CSC groups have the same number of entries in the closed arms $(C A)$ indicating that stress procedure did not affect spontaneous locomotor activity. Data represent mean \pm SEM. $* p<0.001$ versus single-housed $(\mathrm{SHC})$ control mice. SHC $(n=16)$ and CSC $(n=16)$
Bahi et al. 2013b). This has been demonstrated to be an effective protocol for the study of extinction and reinstatement (Bahi 2012). As shown in Fig. 4a, one-way ANOVA revealed that mice initially demonstrated an equivalent amount of time spent in each of the two conditioning chambers $\left(F_{(1,14)}=0.739\right.$, $p=0.404)$. When tested in the post-conditioning session, the mice demonstrated a robust EtOH-CPP that was significantly greater than that of the initial preference $\left(F_{(1,14)}=101.582\right.$, $p<0.0001$ ) (Fig. 4a). More importantly, no difference between SHC and CSC groups was found (stress groups main effect: $F_{(1,14)}=0.880, p=0.364$; interaction between time and stress groups: $F_{(1,14)}=0.780, p=0.784$ ) (Fig. $4 b$ ). During the first day of extinction performed in the absence of ethanol, both SHC $(n=8)$ and CSC $(n=8)$ mice showed a clear initial preference for the chamber previously associated with ethanol (main effect of time: $F_{(1,14)}=118.556, p<0.0001$; main effect of stress: $F_{(1,14)}=0.033, p=0.858$, interaction between time and stress: $\left.F_{(1,14)}=2.061, p=0.173\right)$. However, EtOH-CPP extinction was slower in CSC compared to SHC mice. Statistical analysis
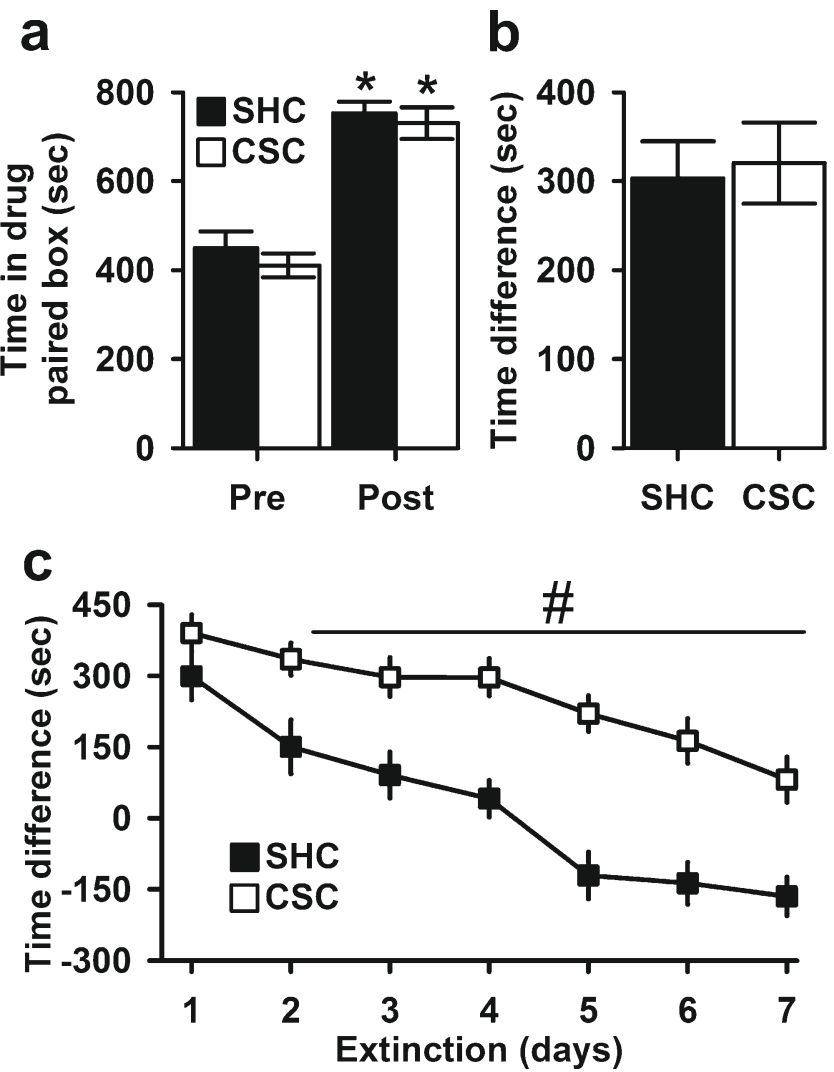

Fig. 4 Chronic psychosocial stress effects on ethanol-induced conditioned place preference "EtOH-CPP" extinction. a Mice were tested in the pre-conditioning test and no side preference was detected. Also, both groups acquired EtOH-CPP as indicated by increased time spent in the drug-paired side during the post-conditioning session. ${ }^{*} p<0.001$ versus pre-conditioning. b The difference between pre- and post-conditioning sessions indicates no significant difference between the stress groups. c CSC mice showed delayed EtOH-CPP extinction. $\# p<0.01$ versus single-housed $(S H C)$ control mice. Data represent mean \pm SEM. SHC $(n=8)$ and $\operatorname{CSC}(n=8)$ 
revealed a significant main effect of time $\left(F_{(6,84)}=35.150\right.$, $p<0.0001)$ and a main effect of stress $\left(F_{(1,14)}=25.606\right.$, $p<0.0001)$. More importantly, the interaction between time and stress was significant $\left(F_{(6,84)}=3.094, p=0.009\right)$ (Fig. $4 \mathrm{c}$ ).

Experiment 2: effects of CSC housing on ethanol-induced reinstatement of EtOH-CPP

As a second experiment, we evaluated the efficacy of subordinate colony housing as a stressor necessary to induce reinstatement in C57BL/6 after a priming injection of ethanol. The results describing the effects of CSC chronic psychosocial stress on ethanol-primed reinstatement of EtOH-CPP behavior are depicted in Fig. 5. A total of 16 mice were evenly and randomly distributed into two groups ( $\mathrm{SHC}, n=8$; CSC, $n=8$ ). The one-way ANOVA test of the pre-conditioning baseline showed that mice spent an almost equal amount of

a

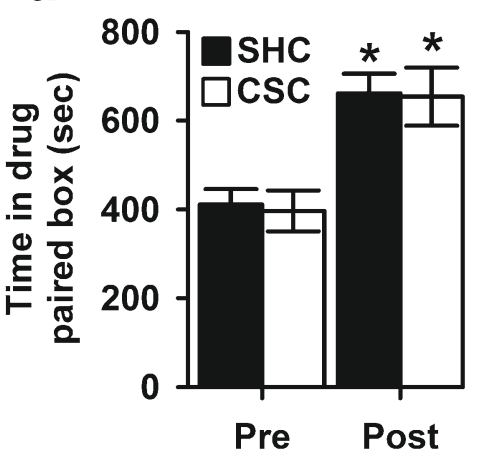

b

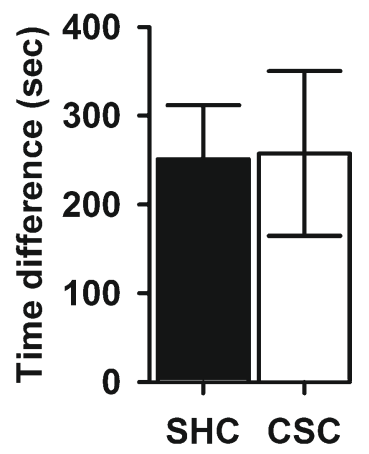

C
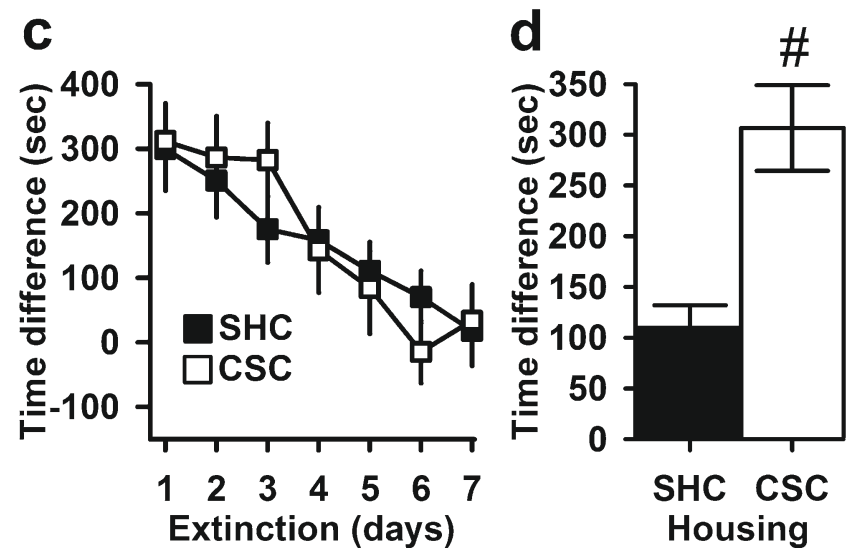

Fig. 5 Chronic psychosocial stress effects on ethanol-induced reinstatement of EtOH-CPP after extinction. a Mice were tested in the preconditioning test and no side preference was detected. Also, both groups acquired EtOH-CPP as indicated by increased time spent in the drugpaired side during the post-conditioning session. ${ }^{*} p<0.001$ versus preconditioning. b The difference between pre- and post-conditioning sessions indicates no difference between the stress groups. $\mathbf{c}$ Both $\mathrm{SHC}$ and CSC mice showed similar EtOH-CPP extinction. d CSC mice showed increased drug-induced reinstatement of EtOH-CPP after extinction. $\# p<0.05$ versus single-housed $(S H C)$ control mice. Data represent mean \pm SEM. SHC $(n=8)$ and CSC $(n=8)$ time in the two chambers. In fact, and as depicted in Fig. 5a, there were no significant differences in the time spent in the two chambers $\left(F_{(1,14)}=0.061, p=0.808\right)$. Thus, the test procedures were considered unbiased in terms of chamber preferences of untreated mice. In addition, both SHC and CSC trained with ethanol acquired significantly higher CPP scores $\left(F_{(1,14)}=21.017, p<0.0001\right)$ (Fig. 5a). More importantly, no apparent difference between the two groups was found $\left(F_{(1,14)}=0.071, p=0.793\right)$ (Fig. 5b). On extinction day 1, the $\mathrm{CPP}$ effects remained. Thus, the animals spent a significantly longer time in the ethanol-paired chamber as compared to the pre-conditioning test $\left(F_{(1,14)}=49.285, p<0.0001\right)$ (Fig. $5 \mathrm{c}$ ), and there was no difference from the post-conditioning test $\left(F_{(1,14)}=1.233, p=0.286\right)$. In contrast, full extinction was observed on extinction day 7 when the ethanol-paired chamber time did not differ from that of the pre-conditioning test (main effect of time: $F_{(6,84)}=11.483, p<0.0001$ ) (Fig. 5c), but both groups extinguished in an equivalent way (main effect of stress groups: $F_{(1,14)}=0.019, p=0.891$ ) (Fig. $5 \mathrm{c}$ ). The next day, the readministration of ethanol at a lower dose of that administered during conditioning $(0.5 \mathrm{~g} / \mathrm{kg})$ resulted in reinstatement of place preference as revealed by the mice spending significantly longer time in the ethanol-paired chamber during the reinstatement test as compared to the pre-conditioning test (main effect of time: $\left.F_{(1,14)}=76.326, p<0.0001\right)$. More importantly and as depicted in Fig. 5d, the interaction between time and stress was significant $\left.F_{(1,14)}=17.064, p=0.001\right)$. To formally establish that a priming dose of alcohol reinstates CPP in CSC mice but not SHC mice, time spent in the drug-paired compartment was compared to the last day of extinction in both groups. A mixed ANOVA with housing history as the between-subjects factor and time as the within-subjects factor showed a main effect of time $\left(F_{(1,14)}=21.469, p<0.0001\right)$ and housing history $\left(F_{(1,14)}=4.252, p=0.058\right)$. More importantly, there was a significant interaction between the time and housing history $\left(F_{(1,14)}=5.283, p=0.037\right)$. Post hoc evaluation showed that following a single injection of ethanol, CSC animals previously demonstrating CPP and exposed to chronic stress show a significant preference for the side previously paired with ethanol. In addition, Fig. 5d shows that SHC mice spent 109.8 $\pm 22.3 \mathrm{~s}$ in the ethanol-paired chamber during reinstatement, which appears to reflect reinstatement, albeit lower than in the CSC group. To further clarify this issue, we performed statistical analyses comparing reinstatement- vs. pre-conditioning values for the SHC group. Thus, a mixed ANOVA showed that a single injection of a low dose of ethanol reinstated the CPP behavior in $\operatorname{CSC}\left(F_{(1,14)}=26.361, p<0.0001\right)$ but not in SHC mice $\left(F_{(1,14)}=4.265, p=0.058\right)$. An additional analysis comparing ethanol-induced reinstatement to the last day of extinction in the SHC and CSC groups revealed that a single injection of a low dose of ethanol reinstated the CPP behavior in $\operatorname{CSC}\left(F_{(1,14)}=26.035, p<0.0001\right)$ but not in SHC mice $\left(F_{(1,14)}=\right.$ $3.261, p=0.092)$. In summary, our results showed that a 
priming injection of ethanol was able to reinstate the EtOHCPP in CSC but not in SHC mice.

\section{Discussion}

The results of the present study demonstrate that chronic psychosocial stress produced long-term effects on EtOHCPP. Indeed, and compared to the SHC control mice, the CSC housing delays extinction and increased ethanolinduced reinstatement of EtOH-CPP behavior. These findings are in general agreement with several studies by Ribeiro and co-workers, in which physical (restraint and tail pinch) and social defeat stressors induced reinstatement of drug-induced CPP (Ribeiro Do Couto et al. 2009; Ribeiro Do Couto et al. 2006). The current results are also in agreement with a different study in which stress in rats reinstates alcohol selfadministration (Funk et al. 2005) and with the findings that a priming injection of ethanol, cocaine, amphetamine, and morphine reinstate drug CPP (DeMarco et al. 2009; Feng et al. 2011; Font et al. 2008; Romieu et al. 2004).

Consequences of CSC on body weight gain and anxiety-like behavior

In agreement with previous reports, we showed that CSC mice exhibited reduced body weight gain which is a wellestablished physiological marker of chronic stress in both mice (Reber et al. 2007; Reber and Neumann 2008; Singewald et al. 2009) and rats (Berton et al. 1998; Nyuyki et al. 2012; Plaznik et al. 1993; Stefanski et al. 2001). A reduction in body weight gain was also observed in acutely defeated animals (Berton et al. 1998). However, stress-induced body weight loss was more severe and longer lasting after CSC, thereby confirming our previous results (Bahi 2013a) that weight gain is differentially affected by acute and chronic social stress. It is noteworthy that subordinate defeated animals had free access to food, thus suggesting that the aforementioned difference in body weight gain probably depended on related differences in the anorexigenic effects of stress.

Studies addressing the behavioral effects of CSC have reported some prolonged anxiogenic consequences (Nyuyki et al. 2012; Reber et al. 2007; Singewald et al. 2009; UscholdSchmidt et al. 2012). Thus, we have tested our SHC and CSC mice in an elevated plus maze [a widely validated model of anxiety, including in our hands (Bahi 2013a, b; Bahi and Dreyer 2012a; Bahi et al. 2009)]. In the present study, a reduced number of entries in the open arms of the elevated plus maze, and decreased time spent therein, were observed in CSC mice compared to SHC controls, thus confirming that the former are more anxious than the latter (Bahi 2013a). These findings are in line with a reduction in exploratory behavior found in a Wistar rat line selectively bred for high anxiety-related behavior
(Salome et al. 2004). This cannot be accounted for by motor differences as the number of entries into the closed arms was similar in SHC and CSC mice suggesting that the observed stress-related behavioral alterations are specific. Furthermore, it should be emphasized that changes in line crossings in the open field (Bahi 2013a, b; Bahi and Dreyer 2012a; Veenema et al. 2008), reflecting basic and spontaneous locomotor activity, were not found in CSC mice. These results suggest that the reduced number of entries and the decreased time spent into the open arms found in CSC, compared to SHC mice, is not due to altered locomotor activity but it is influenced by emotion.

One can argue that single-housed animals are not appropriate as controls for the chronic subordinate stress housing condition. This way, it is impossible to parse out whether it is the group housing or the chronic social stress that are causing the group difference. Singewald and colleagues have addressed this issue in a previous study by using group-housed control mice (four mice per cage for 19 days without a dominant resident) (Singewald et al. 2009). In their study, the authors reported that, like CSC mice, group-housed mice showed less exploratory behavior into the open arms of the EPM test compared with SHC mice. Furthermore, group-housed mice gained less weight during the 19-day period compared with SHC mice (Singewald et al. 2009) indicating that group housing itself is stressful for mice and not an appropriate control for the CSC paradigm. One possible explanation is that one mouse out of the group of four may obtain dominant status and subordinate (aggress) its cage mates. Indeed, it was found that in the group-housed mice, only three out of the four mice showed a reduction in body weight gain comparable to CSC compared with CSC mice (Singewald et al. 2009). Taken together, group housing could not be an adequate control condition for $\mathrm{CSC}$ housing.

\section{Consequences of CSC on extinction and reinstatement of ethanol-CPP}

Consistent with our primary hypothesis and previous research using different stress models, chronic psychosocial stress increased ethanol-induced reinstatement of EtOH-CPP. Specifically, when ethanol $(0.5 \mathrm{~g} / \mathrm{kg})$ was used for priming, CSC mice spent significantly more time in the alcohol-paired chamber relative to SHC controls given the same priming dose.

The finding that chronic psychosocial stress caused reinstatement of ethanol-seeking behavior is novel and extends previous work done by others examining reinstatement of drug self-administration. In these studies, physical stress (foot shock) given for various periods of time reinstated rat leverpressing for ethanol (Liu and Weiss 2003; Martin-Fardon et al. 2000), cocaine (Capriles et al. 2003; Lu et al. 2001), heroin (Leri et al. 2004; Shaham and Stewart 1996; Zhou et al. 2008), and morphine (Mueller et al. 2002). Also, immobilization stress given within the place preference chamber is capable of producing reinstatement of cocaine-seeking behavior in 
male Sprague-Dawley rats (Sanchez et al. 2003). More importantly, few reports using the place conditioning procedure have demonstrated that the restraint stress and forced swim stress reinstated ethanol-induced place preference (Bhutada et al. 2012). Also, increased vulnerability to reinstatement induced by cocaine priming was observed in grouped adult mice exposed to social defeat stressors (Ribeiro Do Couto et al. 2009). Taken together, only animals that experienced defeat in a social interaction with a dominant resident displayed a reinstatement of ethanol-induced CPP, suggesting that chronic social stress is as effective as physical stress in reinstating drug seeking. At the molecular level, it has been reported that the mesocorticolimbic dopamine system was activated following social defeat stress (Burke et al. 2013; Tidey and Miczek 1996; Wand et al. 2007; Yavich and Tiihonen 2000), and therefore this system could be involved in chronic social stress-induced reinstatement of ethanol seeking behavior. In addition, Nikulina and co-workers have reported that within $30 \mathrm{~min}$ after social defeat stress in rats, the expression levels of $\mu$-opioid receptor (MOR)-encoding mRNA, as evaluated and quantified by in situ hybridization, increased in the lateral ventral tegmental area (VTA) (Nikulina et al. 1999). These findings suggest that stress-induced alteration of MOR transcripts level in the VTA may be involved in the consequences of social defeat stress. At the serotoninergic level, chronic social defeat increased $5-\mathrm{HT}_{1 \mathrm{~B}}$ receptor mRNA levels in the rostral nucleus accumbens shell (Furay et al. 2011). This brain region is highly involved in the rewarding properties of drugs of abuse in general and ethanol in particular (Bassareo et al. 2003). Consequently, 5- $\mathrm{HT}_{1 \mathrm{~B}}$ receptor expression increase in this particular region may facilitate chronic social stress-induced susceptibility to the rewarding aspects of ethanol.

Previous work from our laboratory has shown that, using the two-bottle choice drinking paradigm, CSC housing increased voluntary alcohol intake and exacerbated EtOH-CPP acquisition (Bahi 2013a) in mice. Along the same line, Peters and colleagues were able to show that shorter stress periods produced the same effect. In fact, following 14 days of chronic subordinate colony housing, the CSC mice consumed significantly more ethanol than SHC controls (Peters et al. 2013). However, to our knowledge, this is the first study to report that CSC housing-induced chronic psychosocial stress produces reinstatement of ethanol-induced CPP behavior. These results are in agreement with those described previously suggesting that chronic psychosocial stress may interact with drug dependence through the reactivation of EtOH-CPP following a prolonged drug-free period. Nevertheless, it should be emphasized that acute exposure to social defeat decreased ethanol self-administration, reduced rates of responding during extinction, and did not reinstate ethanol-seeking behavior (Funk et al. 2005). The discrepancy between these observations (and, in particular, those of the present experiment) and those from the studies by Funk et al. may be the result of procedural differences. In that study, only five exposures to social defeat were given. In our procedure, however, the four intruder experimental mice were housed for 19 consecutive days with a dominant resident mouse and were transferred to a novel resident cage in days 8 and 15. Thus, the fewer defeat exposure sessions used by Funk et al. may have resulted in weaker reinstatement behavior.

Deroche and colleagues have shown that cross-sensitization may occur between stress and chronic drug taking (Deroche et al. 1992), since stress activates the hypothalamic-pituitaryadrenal axis and induces the secretion of corticosterone. Therefore, when rats were exposed to environmental stressors (foot shock or physical restraint) or social defeat, increased levels of CRF transcripts were found in the shell of the nucleus accumbens, the basolateral and central amygdalar nuclei and the dorsal region of the bed nucleus of the stria terminalis (Funk et al. 2006). More importantly, it has been reported that, when exposed to an acute heterotypic stressor (5 min on an elevated platform) CSC mice have shown an exaggerated adrenal corticosterone response (Fuchsl et al. 2013; Uschold-Schmidt et al. 2012). Also, it has been reported that corticosterone secretion following social isolation stress is required for the expression of the enhanced locomotor response to morphine (Deroche et al. 1994) and amphetamine (Deroche et al. 1993). In addition, Yohimbine, which provokes stress- and anxiety-like behaviors in both humans and rodents, increased operant ethanol self-administration. Yohimbine-induced reinstatement of alcohol seeking was attenuated by the corticotrotropin-releasing factor 1 (CRF1) receptor antagonist antalarmin (Marinelli et al. 2007). More importantly, preclinical evidence with pharmacological therapies targeting CRF receptor (CRF-R) signaling were involved in the regulation of neurobiological responses to ethanol; CRF-R antagonists protect against increased ethanol intake and relapse-like behaviors precipitated by exposure to a stressful event [for review see Lowery and Thiele 2010]. Therefore, the fact that chronic psychosocial stress reinstated drug seeking in animals following an extinction period confirms clinical impressions that stressors can provoke relapse in individuals after long periods of drug abstinence (Breese et al. 2005, 2011). The impact of social stress on initiation and extinction of drug consumption, but also on relapse, was extensively reviewed by Aguilar and colleagues (Aguilar et al. 2013).

Taken together, these data and our present findings suggest that the initial memory formation involved in acquisition (Bahi 2013a), the inhibitory learning unique to extinction, as well as memory retrieval involved in drug-induced reinstatement of EtOH-seeking behavior in mice were affected by CSC housing. Additionally, our experiments showed that etOH reward and the conditioned rewarding effects of etOH were altered by chronic psychosocial stress. In conclusion, comprehension of the multiple molecular and neurochemical events 
involved in chronic psychosocial stress- and drug-induced relapse to alcohol dependence may therefore shed more light on the biological basis of addiction in general and alcoholism in particular.

Acknowledgments The authors would like to acknowledge Ms. Crysta D'souza, Mr. Mohamed Elwasila, and Mr. Mohamed Shafiullah for their technical assistance and Dr. Mahmoud Hag Ali from the Central Animal Facility for his advice on veterinary care. The authors are also grateful to Prof. Keith M. Bagnall for proofreading the manuscript.

Disclosure/conflict of interest The authors declare no conflicts of interests. $\mathrm{AB}$ is receiving salary and funds from the United Arab Emirates University. The funder had no role in the study design, data collection and analysis, decision to publish, or preparation of the manuscript.

\section{References}

Aguilar MA, Garcia-Pardo MP, Montagud-Romero S, Minarro J, Do Couto BR (2013) Impact of social stress in addiction to psychostimulants: what we know from animal models. Curr Pharm Des (in press)

Bahi A (2012) The selective metabotropic glutamate receptor 7 allosteric agonist AMN082 prevents reinstatement of extinguished ethanolinduced conditioned place preference in mice. Pharmacol Biochem Behav 101:193-200

Bahi A (2013a) Increased anxiety, voluntary alcohol consumption and ethanol-induced place preference in mice following chronic psychosocial stress. Stress 16:441-451

Bahi A (2013b) Individual differences in elevated plus-maze exploration predicted higher ethanol consumption and preference in outbred mice. Pharmacol Biochem Behav 105:83-88

Bahi A, Dreyer JL (2012a) Hippocampus-specific deletion of tissue plasminogen activator "tPA" in adult mice impairs depression- and anxiety-like behaviors. Eur Neuropsychopharmacol 22:672-682

Bahi A, Dreyer JL (2012b) Involvement of nucleus accumbens dopamine D1 receptors in ethanol drinking, ethanol-induced conditioned place preference, and ethanol-induced psychomotor sensitization in mice. Psychopharmacol (Berl) 222:141-153

Bahi A, Dreyer JL (2012c) Involvement of tissue plasminogen activator "tPA" in ethanol-induced locomotor sensitization and conditionedplace preference. Behav Brain Res 226:250-258

Bahi A, Mineur YS, Picciotto MR (2009) Blockade of protein phosphatase 2B activity in the amygdala increases anxiety- and depressionlike behaviors in mice. Biol Psychiatry 66:1139-1146

Bahi A, Sadek B, Schwed SJ, Walter M, Stark H (2013a) Influence of the novel histamine $\mathrm{H}$ receptor antagonist ST1283 on voluntary alcohol consumption and ethanol-induced place preference in mice. Psychopharmacol (Berl) 228:85-95

Bahi A, Tolle V, Fehrentz JA, Brunel L, Martinez J, Tomasetto CL, Karam SM (2013b) Ghrelin knockout mice show decreased voluntary alcohol consumption and reduced ethanol-induced conditioned place preference. Peptides 43C:48-55

Bassareo V, De Luca MA, Aresu M, Aste A, Ariu T, Di Chiara G (2003) Differential adaptive properties of accumbens shell dopamine responses to ethanol as a drug and as a motivational stimulus. Eur J Neurosci 17:1465-1472

Berton O, Aguerre S, Sarrieau A, Mormede P, Chaouloff F (1998) Differential effects of social stress on central serotonergic activity and emotional reactivity in Lewis and spontaneously hypertensive rats. Neuroscience 82:147-159
Bhutada P, Mundhada Y, Ghodki Y, Dixit P, Umathe S, Jain K (2012) Acquisition, expression, and reinstatement of ethanol-induced conditioned place preference in mice: effects of exposure to stress and modulation by mecamylamine. J Psychopharmacol 26:315-323

Breese GR, Chu K, Dayas CV, Funk D, Knapp DJ, Koob GF, Le DA, O'Dell LE, Overstreet DH, Roberts AJ, Sinha R, Valdez GR, Weiss F (2005) Stress enhancement of craving during sobriety: a risk for relapse. Alcohol Clin Exp Res 29:185-195

Breese GR, Sinha R, Heilig M (2011) Chronic alcohol neuroadaptation and stress contribute to susceptibility for alcohol craving and relapse. Pharmacol Ther 129:149-171

Burke AR, Forster GL, Novick AM, Roberts CL, Watt MJ (2013) Effects of adolescent social defeat on adult amphetamine-induced locomotion and corticoaccumbal dopamine release in male rats. Neuropharmacology 67:359-369

Capriles N, Rodaros D, Sorge RE, Stewart J (2003) A role for the prefrontal cortex in stress- and cocaine-induced reinstatement of cocaine seeking in rats. Psychopharmacol (Berl) 168:66-74

Chaplin TM, Hong K, Fox HC, Siedlarz KM, Bergquist K, Sinha R (2010) Behavioral arousal in response to stress and drug cue in alcohol and cocaine addicted individuals versus healthy controls. HumPsychopharmacol 25:368-376

Childs E, O'Connor S, de Wit H (2011) Bidirectional interactions between acute psychosocial stress and acute intravenous alcohol in healthy men. Alcohol Clin Exp Res 35:1794-1803

Cunha JM, Masur J (1978) Evaluation of psychotropic drugs with a modified open field test. Pharmacology 16:259-267

DeMarco A, Dalal RM, Pai J, Aquilina SD, Mullapudi U, Hammel C, Kothari SK, Kahanda M, Liebling CN, Patel V, Schiffer WK, Brodie JD, Dewey SL (2009) Racemic gamma vinyl-GABA (R, S-GVG) blocks methamphetamine-triggered reinstatement of conditioned place preference. Synapse 63:87-94

Deroche V, Piazza PV, Casolini P, Le Moal M, Simon H (1993) Sensitization to the psychomotor effects of amphetamine and morphine induced by food restriction depends on corticosterone secretion. Brain Res 611:352-356

Deroche V, Piazza PV, Casolini P, Maccari S, Le Moal M, Simon H (1992) Stress-induced sensitization to amphetamine and morphine psychomotor effects depend on stress-induced corticosterone secretion. Brain Res 598:343-348

Deroche V, Piazza PV, Le Moal M, Simon H (1994) Social isolationinduced enhancement of the psychomotor effects of morphine depends on corticosterone secretion. Brain Res 640:136-139

Enoch MA (2011) The role of early life stress as a predictor for alcohol and drug dependence. Psychopharmacol (Berl) 214:17-31

Feng B, Xing JH, Jia D, Liu SB, Guo HJ, Li XQ, He XS, Zhao MG (2011) Blocking $\alpha 4 \beta 2$ and $\alpha 7$ nicotinic acetylcholine receptors inhibits the reinstatement of morphine-induced CPP by drug priming in mice. Behav Brain Res 220:100-105

Font L, Miquel M, Aragon CM (2008) Involvement of brain catalase activity in the acquisition of ethanol-induced conditioned place preference. Physiol Behav 93:733-741

Fox HC, Talih M, Malison R, Anderson GM, Kreek MJ, Sinha R (2005) Frequency of recent cocaine and alcohol use affects drug craving and associated responses to stress and drug-related cues. Psychoneuroendocrinology 30:880-891

Fuchsl AM, Uschold-Schmidt N, Reber SO (2013) Chronic psychosocial stress in male mice causes an up-regulation of scavenger receptor class B type 1 protein in the adrenal glands. Stress 16:461-468

Funk D, Harding S, Juzytsch W, Le AD (2005) Effects of unconditioned and conditioned social defeat on alcohol self-administration and reinstatement of alcohol seeking in rats. Psychopharmacology 183:341-349

Funk D, Li Z, Le AD (2006) Effects of environmental and pharmacological stressors on c-fos and corticotropin-releasing factor mRNA in rat brain: Relationship to the reinstatement of alcohol seeking. Neuroscience 138:235-243 
Furay AR, McDevitt RA, Miczek KA, Neumaier JF (2011) 5-HT1B mRNA expression after chronic social stress. Behav Brain Res 224:350-357

Heilig M, Egli M (2006) Pharmacological treatment of alcohol dependence: target symptoms and target mechanisms. Pharmacol Ther $111: 855-876$

Jennison KM (1992) The impact of stressful life events and social support on drinking among older adults: a general population survey. Int $\mathrm{J}$ Aging Hum Dev 35:99-123

Joffe JM, Rawson RA, Mulick JA (1973) Control of their environment reduces emotionality in rats. Science 180:1383-1384

Kosten TR, Rounsaville BJ, Kleber HD (1986) A 2.5-year follow-up of depression, life crises, and treatment effects on abstinence among opioid addicts. Archives Gen Psychiatry 43:733-738

Krishnan S, Nash JF Jr, Maickel RP (1991) Free-choice ethanol consumption by rats: effects of ACTH4-10. Alcohol 8:401-404

Leri F, Tremblay A, Sorge RE, Stewart J (2004) Methadone maintenance reduces heroin- and cocaine-induced relapse without affecting stressinduced relapse in a rodent model of poly-drug use. Neuropsychopharmacology 29:1312-1320

Lipton R (1997) The relationship between alcohol, stress, and depression in Mexican Americans and non-Hispanic whites. Behav Med 23:101-111

Liu X, Weiss F (2003) Stimulus conditioned to foot-shock stress reinstates alcohol-seeking behavior in an animal model of relapse. Psychopharmacol (Berl) 168:184-191

Lowery EG, Thiele TE (2010) Pre-clinical evidence that corticotropinreleasing factor $(\mathrm{CRF})$ receptor antagonists are promising targets for pharmacological treatment of alcoholism. CNS Neurol Disord Drug Targets 9:77-86

Lu L, Liu D, Ceng X (2001) Corticotropin-releasing factor receptor type 1 mediates stress-induced relapse to cocaine-conditioned place preference in rats. Eur J Pharmacol 415:203-208

Marinelli PW, Funk D, Juzytsch W, Harding S, Rice KC, Shaham Y, Le AD (2007) The CRF1 receptor antagonist antalarmin attenuates yohimbine-induced increases in operant alcohol self-administration and reinstatement of alcohol seeking in rats. Psychopharmacol (Berl) 195:345-355

Martin-Fardon R, Ciccocioppo R, Massi M, Weiss F (2000) Nociceptin prevents stress-induced ethanol-but not cocaine-seeking behavior in rats. Neuroreport 11:1939-1943

Mueller D, Perdikaris D, Stewart J (2002) Persistence and drug-induced reinstatement of a morphine-induced conditioned place preference. Behav Brain Res 136:389-397

Nash JF Jr, Maickel RP (1988) The role of the hypothalamic-pituitaryadrenocortical axis in post-stress induced ethanol consumption by rats. Prog in Neuro-psychopharmacol Biol Psychiatry 12:653-671

Nikulina EM, Hammer RP Jr, Miczek KA, Kream RM (1999) Social defeat stress increases expression of mu-opioid receptor mRNA in rat ventral tegmental area. Neuroreport 10:3015-3019

Nyuyki KD, Beiderbeck DI, Lukas M, Neumann ID, Reber SO (2012) Chronic subordinate colony housing (CSC) as a model of chronic psychosocial stress in male rats. PLoS One 7:e52371

Peters S, Slattery DA, Flor PJ, Neumann ID, Reber SO (2013) Differential effects of baclofen and oxytocin on the increased ethanol consumption following chronic psychosocial stress in mice. Addict Biol 18:66-77

Plaznik A, Palejko W, Stefanski R, Kostowski W (1993) Open field behavior of rats reared in different social conditions: the effects of stress and imipramine. Pol J Pharmacol 45:243-252

Reber SO, Birkeneder L, Veenema AH, Obermeier F, Falk W, Straub RH, Neumann ID (2007) Adrenal insufficiency and colonic inflammation after a novel chronic psycho-social stress paradigm in mice: implications and mechanisms. Endocrinology 148:670-682
Reber SO, Neumann ID (2008) Defensive behavioral strategies and enhanced state anxiety during chronic subordinate colony housing are accompanied by reduced hypothalamic vasopressin, but not oxytocin, expression. Ann N Y Acad Sci 1148:184-195

Ribeiro Do Couto B, Aguilar MA, Lluch J, Rodriguez-Arias M, Minarro $\mathrm{J}$ (2009) Social experiences affect reinstatement of cocaine-induced place preference in mice. Psychopharmacol (Berl) 207:485-498

Ribeiro Do Couto B, Aguilar MA, Manzanedo C, Rodriguez-Arias M, Armario A, Minarro J (2006) Social stress is as effective as physical stress in reinstating morphine-induced place preference in mice. Psychopharmacol (Berl) 185:459-470

Romieu P, Meunier J, Garcia D, Zozime N, Martin-Fardon R, Bowen WD, Maurice T (2004) The sigmal (sigma1) receptor activation is a key step for the reactivation of cocaine conditioned place preference by drug priming. Psychopharmacol (Berl) 175:154-162

Salome N, Salchner P, Viltart O, Sequeira H, Wigger A, Landgraf R, Singewald N (2004) Neurobiological correlates of high (HAB) versus low anxiety-related behavior (LAB): differential Fos expression in HAB and LAB rats. Biol Psychiatry 55:715-723

Sanchez CJ, Bailie TM, Wu WR, Li N, Sorg BA (2003) Manipulation of dopamine d1-like receptor activation in the rat medial prefrontal cortex alters stress- and cocaine-induced reinstatement of conditioned place preference behavior. Neuroscience 119:497-505

Shaham Y, Stewart J (1996) Effects of opioid and dopamine receptor antagonists on relapse induced by stress and re-exposure to heroin in rats. Psychopharmacol (Berl) 125:385-391

Singewald GM, Nguyen NK, Neumann ID, Singewald N, Reber SO (2009) Effect of chronic psychosocial stress-induced by subordinate colony (CSC) housing on brain neuronal activity patterns in mice. Stress 12:58-69

Sinha R (2001) How does stress increase risk of drug abuse and relapse? Psychopharmacol (Berl) 158:343-359

Sinha R (2007) The role of stress in addiction relapse. Curr Psychiatry Rep 9:388-395

Sinha R, Fuse T, Aubin LR, O'Malley SS (2000) Psychological stress, drug-related cues and cocaine craving. Psychopharmacol (Berl) 152:140-148

Stefanski V, Knopf G, Schulz S (2001) Long-term colony housing in Long Evans rats: immunological, hormonal, and behavioral consequences. J Neuroimmunol 114:122-130

Tidey JW, Miczek KA (1996) Social defeat stress selectively alters mesocorticolimbic dopamine release: an in vivo microdialysis study. Brain Res 721:140-149

Tsigos C, Chrousos GP (2002) Hypothalamic-pituitary-adrenal axis, neuroendocrine factors and stress. J Psychosom Res 53:865-871

Uschold-Schmidt N, Nyuyki KD, Fuchsl AM, Neumann ID, Reber SO (2012) Chronic psychosocial stress results in sensitization of the HPA axis to acute heterotypic stressors despite a reduction of adrenal in vitro ACTH responsiveness. Psychoneuroendocrinology 37:1676-1687

Veenema AH, Reber SO, Selch S, Obermeier F, Neumann ID (2008) Early life stress enhances the vulnerability to chronic psychosocial stress and experimental colitis in adult mice. Endocrinology 149:2727-2736

Volpicelli JR (1987) Uncontrollable events and alcohol drinking. Br J Addict 82:381-392

Waltman C, Blevins LS Jr, Boyd G, Wand GS (1993) The effects of mild ethanol intoxication on the hypothalamic-pituitary-adrenal axis in nonalcoholic men. J Clin Endocrinol Metab 77:518-522

Wand GS, Dobs AS (1991) Alterations in the hypothalamic-pituitaryadrenal axis in actively drinking alcoholics. J Clin Endocrinol Metab 72:1290-1295

Wand GS, Oswald LM, McCaul ME, Wong DF, Johnson E, Zhou Y, Kuwabara H, Kumar A (2007) Association of amphetamine- 
induced striatal dopamine release and cortisol responses to psychological stress. Neuropsychopharmacology 32:2310-2320

Yavich L, Tiihonen J (2000) Ethanol modulates evoked dopamine release in mouse nucleus accumbens: dependence on social stress and dose. Eur J Pharmacol 401:365-373

Zhou Y, Leri F, Cummins E, Hoeschele M, Kreek MJ (2008) Involvement of arginine vasopressin and $\mathrm{V} 1 \mathrm{~b}$ receptor in heroin withdrawal and heroin seeking precipitated by stress and by heroin. Neuropsychopharmacology 33:226-236

Zimmermann U, Spring K, Kunz-Ebrecht SR, Uhr M, Wittchen HU, Holsboer F (2004) Effect of ethanol on hypothalamicpituitary-adrenal system response to psychosocial stress in sons of alcohol-dependent fathers. Neuropsychopharmacology 29:1156-1165 